\title{
Heart rate recovery and blood pressure response during exercise testing in patients with microvascular angina
}

\author{
Bong-Joon Kim¹, Eun-Ah Jo², Sung-II Im¹, Hyun-Su Kim¹, Jung Ho Heo ${ }^{1}$ and Kyoung-Im Cho ${ }^{1 *}$ (D)
}

\begin{abstract}
Background: Angina pectoris with a normal coronary angiogram, termed microvascular angina (MVA), is an important clinical entity; however, its causes remain unclear. Autonomic dysfunction is one of the possible causes. Therefore, this study aimed to investigate parasympathetic dysfunction assessed by heart rate recovery (HRR) and increased sympathetic activity assessed by exaggerated blood pressure (BP) response (EBPR) to exercise in MVA.

Methods: The study participants were consecutive patients with anginal chest pain who underwent both coronary angiography with an ergonovine provocation test and a treadmill exercise test between January 2008 and February 2015. Patients with significant coronary artery disease (coronary artery stenosis $\geq 50 \%$ ) or significant coronary artery spasm ( $\geq 90 \%$ ) were excluded. Based on the treadmill exercise test, patients were categorized into the microvascular angina (MVA) group (patients with uniform ST depression $\geq 1 \mathrm{~mm}$ ) and the control group. HRR was defined as peak heart rate minus heart rate after a 1 min recovery; blunted HRR was defined as $\leq 12$ beats/min. EBPR was defined as a peak exercise systolic BP $\geq 210 \mathrm{mmHg}$ in men and $\geq 190 \mathrm{mmHg}$ in women. These parameters were compared between patients with MVA and the controls.
\end{abstract}

Results: Among the 970 enrolled patients (mean age 53.1 years; female 59.0\%), 191 (20.0\%) were diagnosed with MVA. In baseline characteristics, the MVA group had older participants, female predominance, and a higher prevalence of hypertension. The MVA group showed significantly lower HRR 1 min (24.9 \pm 15.9 vs. $31.3 \pm 22.7, p<0$. 001) compared with the control group. Moreover, the proportion of EBPR was significantly higher in the MVA group than in the control group $(21.5 \%$ vs. $11.6 \%, \mathrm{p}<0.001)$. Multivariable logistic regression analysis showed that age (odds ratio (OR), 1.045; 95\% confidence interval (Cl), 1.026-1.064; $\mathrm{p}<0.001)$, HRR 1 min (OR, 0.990; 95\% Cl, 0. 982-0.999; $p=0.022)$, and EBPR (OR, 1.657; 95\% Cl, 1.074-2.554; $p=0.022)$ were independently associated with MVA.

Conclusion: HRR and EBPR were associated with MVA, which suggests a link between MVA and autonomic dysregulation.

Keywords: Microvascular angina, Cardiac autonomic function, Heart rate recovery

\section{Introduction}

Microvascular angina (MVA) is a clinical condition characterized by exertional angina, exercise-induced myocardial ischemia, and a normal coronary angiogram [1]. Despite the absence of significant coronary stenosis, MVA shows evidence of myocardial ischemia on stress tests [2]. In addition, quality of life in patients with MVA

\footnotetext{
*Correspondence: kyoungim74@gmail.com

'Division of Cardiology, Department of Internal Medicine, Kosin University

College of Medicine, 34, Amnam-dong, Seo-gu, Busan 602-702, Korea

Full list of author information is available at the end of the article
}

is often impaired by long-term, recurrent chest pain [3], and MVA may affect left ventricular systolic and diastolic function [4]. The precise mechanism remains unclear, and microvascular dysfunction, autonomic dysregulation, generalized vascular disorder, and abnormal subendocardial perfusion have been suggested [5]. Among them, autonomic dysfunction is one of the major possible causes of vasomotion in MVA [6].

An exercise treadmill test, easy to apply in clinical practice, can detect the presence of myocardial ischemia and changes in heart rate and blood pressure (BP)

(C) The Author(s). 2019 Open Access This article is distributed under the terms of the Creative Commons Attribution 4.0 International License (http://creativecommons.org/licenses/by/4.0/), which permits unrestricted use, distribution, and 
during exercise. Heart rate recovery (HRR) is a simple noninvasive measurement related to autonomic nervous system dysfunction that indicates impaired parasympathetic reactivation [7-9]. Previous studies have shown that blunted HRR, defined as a $\leq 12$ beats/min decrease in heart rate (HR) from peak exercise to $1 \mathrm{~min}$ into recovery, is a powerful predictor of overall mortality $[7,10]$.

Blood pressure response during graded exercise is a useful parameter to predict hypertension, [11] and is associated with adverse cardiovascular outcomes [12, 13]. In particular, exaggerated BP response (EBPR) to exercise is related to impaired endothelial vasodilator function [14] and is also thought to be a predictable marker of masked hypertension (MHT) [15].

Therefore, this study aimed to investigate autonomic dysfunction as assessed by HRR and increased sympathetic activity as assessed by EBPR in MVA.

\section{Methods}

This is cross-sectional, observational, single-center cohort study. We retrospectively reviewed a total of 1393 patients without significant coronary artery disease (coronary artery stenosis $250 \%$ ) who underwent both CAG with ergonovin provocation test and treadmill test during between January 2007 and August 2015. We excluded the 300 patients with significant coronary spasm $(\geq 90 \%)$ on ergonovin provocation test and 29 patients with other exclusion criteria as follows; any systemic disease such as significant liver disease, neurologic disorder, or malignant disease; secondary hypertension; history of heart failure; valvular heart disease; atrial fibrillation. In enrolled 998 patients, 28 patients were excluded due to outlier value in statistical analysis. MVA was defined as follows: (1) typical stable angina induced by effort, (2) a flat or downsloping depression of the ST segment $>0.1$ $\mathrm{mV}$ below baseline and lasting longer than $0.08 \mathrm{~s}$ during the treadmill test, and (3) normal or near normal coronary arteries on coronary angiogram (vascular wall irregularities or discrete very mild stenosis $(<20 \%)$ ) [16]. Demographic characteristics recorded at the first visit comprised age, sex, height, weight, current medications, smoking history, and other diseases. Blood samples were drawn for measurement of total serum cholesterol, triglycerides, high-density lipoprotein and low-density lipoprotein cholesterol, blood glucose, creatinine, uric acid, and high sensitivity $\mathrm{C}$-reactive protein. Body mass index (BMI) was calculated as the ratio of dry weight in kilograms to height in square meters.

\section{Echocardiographic measurement}

Standard 2-dimensional echocardiography was performed on all subjects lying in the left lateral decubitus position using a $3.5-\mathrm{MHz}$ transducer (Philips iE33, Philips Medical Systems, Bothell, WA, USA), and the echocardiography examiners were blinded to patient information. Measurements of the diameter of the left ventricle (LV) cavity, the thickness of the interventricular septum and posterior wall, and the LV mass index (LVMI) were performed according to criteria outlined by the American Society of Echocardiography [17]. Pulsed wave Doppler of transmitral LV inflow was performed in the apical four-chamber view, with the sample volume placed at the level of the mitral valve tips; Doppler variables were analyzed during three consecutive beats. The following measurements of global LV diastolic function were determined: peak early (E) and late (A) diastolic mitral flow velocity, their ratio $\mathrm{E} / \mathrm{A}$, and early (Ea) diastolic mitral annular velocity.

\section{Exercise treadmill testing}

All patients underwent symptom-limited exercise stress testing (GE CASE T2100; GE Medical Systems, Milwaukee, WI, USA) according to the protocol by Bruce et al. [18]. BP was measured using an automated BP monitor (Suntech Tango; Suntech Medical, Morrisville, NC, USA) throughout the treadmill test using the same arm as was used to measure resting BP. Twelve-lead electrocardiography was monitored continuously and printed at a paper speed of $25 \mathrm{~mm} / \mathrm{s}$; measurements of $\mathrm{HR}$ and $\mathrm{BP}$ were recorded at the end of each 3-min stage, at peak exercise, and at 1-min and 2-min intervals throughout recovery. The participants continued to exercise until volitional fatigue or if HR exceeded 95\% of estimated maximal HR (220 bpm, age). The total exercise time was also recorded. Functional capacity was estimated in metabolic equivalents (METs) on the basis of the speed and grade of the treadmill [19]. During the recovery phase, the subjects continued to walk for $60 \mathrm{~s}$ at a speed of $1.5 \mathrm{mph}$, and then they sat down for $3 \mathrm{~min}$ with continued monitoring of $\mathrm{BP}, \mathrm{HR}$, and heart rhythm. HRR was defined as decrease in HR from peak exercise to 1 min and 2 min after cessation of exercise.

\section{Ergonovine test for provocation of coronary spasm}

After diagnostic coronary angiography, incremental doses of ergonovine were injected intravenously (50, $100,200 \mu \mathrm{g}$ ) over $10 \mathrm{~s}$ [20]. Two minutes after each injection, the coronary angiogram, electrocardiogram, BP, and patient symptoms were assessed. Coronary spasm was defined as near total or localized spasm $(\geq 90 \%$ diameter) of the focal epicardial coronary artery with signs of chest pain or ischemic ST changes according to the Guidelines for Diagnosis and Treatment of Patients with Vasospastic Angina [21]. Intracoronary injection of isosorbide dinitrate was performed on completion of the ergonovine test, regardless of whether or not coronary spasm was confirmed. 


\section{Statistical analysis}

Statistical analyses were performed using the commercially available computer program SPSS 18.0 for Windows (IBM, Chicago, IL, USA). Data are presented as mean \pm standard deviation for continuous variables and as percentages (\%) if the data are categorical. The Mann-Whitney $U$ test was used for continuous variables, and the Chi-square test was used for categorical data. The normality of data was tested using the Kolmogorov-Smirnov test. To identify independent contributors for MVA, univariate and multivariate logistic regression analyses were performed. A $p$-value $<0.05$ was considered statistically significant.

\section{Results}

A total of 970 patients were enrolled; the mean age was 53.1 years, and 59.0\% were female. Among them, 191 were diagnosed with MVA, documented by no significant coronary stenosis with a positive exercise test, and 779 patients with a negative exercise test were compared as controls. In baseline characteristics, the MVA group was older, had a higher proportion of female participants, and had higher prevalence of hypertension and dyslipidemia. No significant differences were observed in BMI, office systolic/diastolic BP, and use of renin-angiotensin system blocker, beta blockers, calcium channel blockers, and statins (Table 1). Laboratory tests showed no significant differences between groups
(Table 2). In echocardiogram parameters, the MVA group showed higher LV septal wall thickness, LV mass index, LA diameter, LA volume, and Ea compared with the control group (Table 3 ).

Table 4 summarizes the results of the exercise test. The MVA group showed significantly lower exercise time, METs, HRR 1 min $(24.9 \pm 15.9$ vs $31.3 \pm 22.7, p$ $<0.001)$, and HRR 2 min (64.1 \pm 17.5 vs. $68.1 \pm 15.5, p=$ $0.004)$ compared with the control group. Moreover, no differences were observed in resting systolic and diastolic BP between groups; however, the proportion of EBPR was significantly higher in the MVA group compared to the control group (21.5 vs. $11.6 \%, \mathrm{p}<0.001)$. Multivariable logistic regression analysis showed that age (odds ratio (OR), 1.045; 95\% confidence interval (CI), 1.026-1.064; $\mathrm{p}<0.001)$, HRR $1 \mathrm{~min}(\mathrm{OR}, 0.990 ; 95 \% \mathrm{CI}$, $0.982-0.999 ; p=0.022$ ), and EBPR (OR, $1.657 ; 95 \% \mathrm{CI}$, $1.074-2.554 ; \mathrm{p}=0.022)$ were independently associated with MVA (Table 5).

\section{Discussion}

The major findings obtained from this study were as follows: (1) HRR for both 1 and 2 min was lower in patients with MVA, (2) the EBPR proportion was higher in the MVA group, and (3) HRR for 1 min and EBPR were independent predictors of MVA. These findings suggest a possible link between MVA and autonomic dysregulation.

Table 1 Baseline characteristics

\begin{tabular}{|c|c|c|c|}
\hline & Control $(n=779)$ & $\operatorname{MVA}(n=191)$ & $p$-value \\
\hline Age, years & $52.0 \pm 10.9$ & $57.7 \pm 9.1$ & $<0.001$ \\
\hline Female gender, n (\%) & $440(56.5)$ & $132(69.1)$ & 0.001 \\
\hline Body mass index, $\mathrm{kg} / \mathrm{m}^{2}$ & $23.3 \pm 6.0$ & $24.0 \pm 6.6$ & 0.221 \\
\hline Systolic BP, mmHg & $127.1 \pm 18.4$ & $129.1 \pm 16.6$ & 0.262 \\
\hline Diastolic BP, mmHg & $78.8 \pm 12.6$ & $78.0 \pm 12.4$ & 0.514 \\
\hline Smoking, n (\%) & $133(25.5)$ & $24(17.9)$ & 0.070 \\
\hline Alcohol, n (\%) & $179(34.4)$ & $30(22.4)$ & 0.009 \\
\hline Hypertension, n (\%) & $141(26.7)$ & $57(37.3)$ & 0.019 \\
\hline Diabetes mellitus, n (\%) & $80(14.4)$ & $27(19.7)$ & 0.146 \\
\hline Dyslipidemia, n (\%) & $212(40.1)$ & $69(51.9)$ & 0.018 \\
\hline Hyperthyroidism, n (\%) & $51(9.6)$ & $9(6.8)$ & 0.510 \\
\hline \multicolumn{4}{|l|}{ Medication } \\
\hline Aspirin, n (\%) & $117(15.0)$ & $34(17.8)$ & 0.422 \\
\hline RAS blocker, n (\%) & $91(11.7)$ & $33(17.3)$ & 0.110 \\
\hline Beta blocker, n (\%) & $69(8.9)$ & $25(13.1)$ & 0.141 \\
\hline Calcium channel blocker, n (\%) & $97(12.5)$ & $31(16.2)$ & 0.314 \\
\hline Diuretics, n (\%) & $21(2.7)$ & $6(3.1)$ & 0.769 \\
\hline Nitrates, n (\%) & 79 (10.1) & $17(8.9)$ & 0.871 \\
\hline Statin, n (\%) & 83 (10.7) & $22(11.5)$ & 0.218 \\
\hline
\end{tabular}

Data are presented as mean \pm SD or frequency with percentage in parenthesis. MVA microvascular angina, BP blood pressure, RAS renin-angiotensin system 
Table 2 Laboratory test

\begin{tabular}{llll}
\hline & Control $(\mathrm{n}=779)$ & MVA $(\mathrm{n}=191)$ & $p$-value \\
\hline Hemoglobin, $\mathrm{g} / \mathrm{dL}$ & $13.5 \pm 4.4$ & $13.4 \pm 2.5$ & 0.695 \\
White blood cells, $10^{3} / \mathrm{\mu L}$ & $7.3 \pm 3.7$ & $7.1 \pm 2.3$ & 0.611 \\
Platelets, $10^{3} / \mathrm{\mu L}$ & $221.6 \pm 56.1$ & $216.8 \pm 55.7$ & 0.291 \\
Uric acid, $\mathrm{mg} / \mathrm{L}$ & $5.4 \pm 1.9$ & $5.2 \pm 1.5$ & 0.374 \\
Creatinine, $\mathrm{mg} / \mathrm{dL}$ & $0.8 \pm 0.2$ & $0.8 \pm 0.8$ & 0.083 \\
Fasting glucose, mg/dL & $100.1 \pm 23.2$ & $101.0 \pm 24.2$ & 0.674 \\
Total cholesterol, mg/dL & $175.7 \pm 36.2$ & $178.9 \pm 38.0$ & 0.315 \\
LDL cholesterol, mg/dL & $104.3 \pm 48.6$ & $104.0 \pm 32.1$ & 0.942 \\
HDL cholesterol, mg/dL & $47.9 \pm 13.3$ & $49.4 \pm 16.2$ & 0.221 \\
Triglycerides, mg/dL & $128.2 \pm 119.8$ & $119.0 \pm 58.7$ & 0.327 \\
hs-CRP, mg/dL & $0.3 \pm 0.9$ & $0.2 \pm 1.0$ & 0.384 \\
TSH, ulU/mL & $2.6 \pm 7.4$ & $2.2 \pm 2.5$ & 0.682 \\
Free T4, ng/dL & $1.5 \pm 5.3$ & $1.2 \pm 0.7$ & 0.649 \\
T3, ng/dL & $94.8 \pm 30.8$ & $91.7 \pm 24.7$ & 0.348 \\
\hline
\end{tabular}

Data are presented as mean \pm SD. MVA microvascular angina, $L D L$ low density lipoprotein, CRP C-reactive protein, TSH thyroid stimulating hormone

Although the mechanism of MVA remains unclear and the terminology is confusing, studies have shown association with autonomic dysfunction. Lanza et al. demonstrated that metaiodobenzylguanidine (MIBI) uptake score is higher in patients with syndrome $\mathrm{X}$, and impairment in cardiac MIBI uptake is associated with a reduction in HR variability indices [22]. Parasympathetic reactivation is an important factor in predicting autonomic dysfunction; as the principal determinant of the decrease in HR during early recovery, this mechanism is independent of age and exercise intensity [23]. Given the

Table 3 Parameters of Echocardiogram

\begin{tabular}{llll}
\hline & Control $(n=779)$ & MVA $(n=191)$ & $p$-value \\
\hline LVEDD, mm & $45.5 \pm 4.6$ & $46.0 \pm 4.6$ & 0.550 \\
LVESD, mm & $28.3 \pm 4.3$ & $28.0 \pm 4.4$ & 0.388 \\
IVSTd, mm & $11.2 \pm 2.0$ & $11.9 \pm 2.3$ & 0.001 \\
PWTd, mm & $10.0 \pm 1.7$ & $10.3 \pm 1.6$ & 0.100 \\
LVMI, g/m ${ }^{2}$ & $112.2 \pm 37.2$ & $121.6 \pm 40.9$ & 0.011 \\
LV EF, \% & $67.9 \pm 7.7$ & $69.4 \pm 7.9$ & 0.049 \\
LA diameter, mm & $34.0 \pm 5.4$ & $35.3 \pm 5.7$ & 0.013 \\
LA volume, mL & $16.5 \pm 5.4$ & $19.8 \pm 7.3$ & $<0.001$ \\
E velocity, cm/sec & $0.7 \pm 0.2$ & $0.7 \pm 0.2$ & 0.104 \\
A velocity, cm/sec & $0.6 \pm 0.2$ & $0.7 \pm 0.2$ & $<0.001$ \\
Ea, cm/sec & $0.08 \pm 0.03$ & $0.07 \pm 0.02$ & 0.005 \\
E/Ea & $9.1 \pm 3.2$ & $10.3 \pm 3.3$ & $<0.001$ \\
\hline Alvalues are & &
\end{tabular}

All values are presented as the mean \pm SD. MVA microvascular angina, LVEDD left ventricular end-diastolic diameter, LVESD left ventricular end-systolic diameter, IVSTd diastolic interventricular septal wall thickness, PWTd diastolic posterior wall thickness, $L V M I$ left ventricular mass index, $R W T$ relative wall thickness, $E F$ ejection fraction, $L A$ left atrial diameter, $E$ peak early diastolic mitral filling velocity, Ea mitral septal annular velocity, $A$ peak late diastolic mitral filling velocity
Table 4 Parameters of Exercise test

\begin{tabular}{llll}
\hline & Control $(n=779)$ & MVA $(n=191)$ & $p$-value \\
\hline Exercise time, min & $7.9 \pm 2.0$ & $6.7 \pm 2.4$ & $<0.001$ \\
Metabolic equivalents & $9.8 \pm 3.3$ & $8.8 \pm 5.2$ & 0.001 \\
Rest heart rate, bpm & $69.5 \pm 12.4$ & $67.3 \pm 11.6$ & 0.028 \\
Max heart rate, bpm & $153.6 \pm 18.6$ & $144.7 \pm 21.7$ & $<0.001$ \\
HR at recovery 1 min, bpm & $122.3 \pm 26.4$ & $119.8 \pm 25.7$ & 0.238 \\
HR at recovery 2 min, bpm & $85.8 \pm 15.1$ & $81.0 \pm 14.3$ & $<0.001$ \\
HRR 1 min, bpm & $31.3 \pm 22.7$ & $24.9 \pm 15.9$ & $<0.001$ \\
HRR 2 min, bpm & $68.1 \pm 15.5$ & $64.1 \pm 17.5$ & 0.004 \\
Blunted HRR, $\mathrm{n}(\%)$ & $236(30.3)$ & $53(27.7)$ & 0.537 \\
Rest systolic BP, mmHg & $126.5 \pm 38.5$ & $127.5 \pm 14.2$ & 0.731 \\
Rest diastolic BP, mmHg & $75.2 \pm 10.8$ & $74.5 \pm 11.2$ & 0.428 \\
Max systolic BP, mmHg & $171.4 \pm 19.8$ & $176.9 \pm 23.9$ & 0.004 \\
Max diastolic BP, mmHg & $81.1 \pm 11.8$ & $83.7 \pm 11.7$ & 0.007 \\
EBPR, n (\%) & $90(11.6)$ & $41(21.5)$ & $<0.001$
\end{tabular}

Data are presented as mean \pm SD or frequency with percentage in parenthesis. MVA microvascular angina, $H R$ heart rate, $H R R$ heart rate recovery, $B P$ blood pressure, EBPR exaggerated blood pressure response

prognostic significance of diminished parasympathetic tone at rest, post-exercise HRR offers a noninvasive and feasible method to assess parasympathetic activation [23, 24]. Reduced HRR after exercise is a powerful predictor of overall mortality in patients without a history of heart failure or coronary revascularization [10]. Because it is simple to calculate from data obtained from standard exercise tests and does not require either 24-h Holter monitoring or specialized baroreflex sensitivity testing, HRR may be useful for assessment of risk in routine clinical practice.

Sympathetic activity is also an important factor of autonomic function, and BP response during exercise is an especially useful parameter. We observed that the MVA group had a higher prevalence of EBPR, suggesting that MVA might have impaired the vascular response. Vascular resistance is involved with multiple factors such as atherosclerosis, vessel spasms, and endothelial dysfunction. BP reflect the change in cardiac output during exercise or recovery can lead to changes in systolic BP [25]. Cardiovascular reactivity to both isometric and dynamic exercise has been shown to be one of the most important markers for predicting hypertension [11]. Impaired vascular function, including increased arterial stiffness and abnormal endothelial function, is associated with increased exercise BP response [12]. Among the parameters of $\mathrm{BP}$ response, exercise $\mathrm{BP}$ response is an important marker of cardiovascular risk that is associated with cardiovascular mortality [26, 27]. In particular, exaggerated exercise BP is a significant predictor for total cardiovascular events and for new onset of resting hypertension [27]. In our data, the MVA group had a 
Table 5 Risk factors associated with the microvascular angina according to logistic regression models

\begin{tabular}{|c|c|c|c|c|}
\hline \multirow[b]{2}{*}{ Risk factors } & \multicolumn{2}{|l|}{ Univariable } & \multicolumn{2}{|l|}{ Multivariable } \\
\hline & Odds ratio $(95 \% \mathrm{Cl})$ & $p$-value & Odds ratio $(95 \% \mathrm{Cl})$ & $p$-value \\
\hline Age & 1.058 (1.040 to 1.076$)$ & $<0.001$ & 1.045 (1.026 to 1.064$)$ & $<0.001$ \\
\hline Female gender & 1.724 (1.229 to 2.417 ) & 0.002 & 1.387 (0.964 to 1.995$)$ & 0.078 \\
\hline HRR 1 min & 0.986 (0.978 to 0.993 ) & $<0.001$ & 0.990 (0.982 to 0.999$)$ & 0.022 \\
\hline $\mathrm{HRR} 2 \mathrm{~min}$ & 0.983 (0.972 to 0.994) & 0.002 & 0.992 (0.980 to 1.003$)$ & 0.154 \\
\hline EBPR & 2.093 (1.390 to 3.151$)$ & $<0.001$ & 1.657 (1.074 to 2.554$)$ & 0.022 \\
\hline
\end{tabular}

$H R R$ heart rate recovery, EBPR exaggerated blood pressure response

higher prevalence of hypertension and higher LV septum thickness and LV mass index compared with the control group. Patients with hypertension or LVH may have frequent BP elevations due to underlying vascular dysfunction to compensate for increased cardiac output in low to moderate exercise [12]. So, we think these factors have affected our results somewhat.

Coronary spasm is one of the major components of chest pain in patients without coronary artery stenosis and it accounts for a large number of unexplained case. Vagal withdrawal is often a component of the mechanisms leading to spontaneous coronary vasospasm [28]. Decreased parasympathetic nervous activity with enhanced sympathetic nervous activity at night is reported to be involved in the mechanism underlying multi-vessel coronary spasm [29]. It is of note that we excluded patients with coronary artery spasm based on the ergonovine provocation test to rule out the effect of coronary spasm. Therefore, our findings demonstrated that, even after the effect of coronary spasm was excluded, autonomic dysfunction remained one of the important mechanisms of MVA.

\section{Limitations}

This study has several important limitations. First, in baseline characteristics, the participants in the MVA group were older, more likely to be female, and had higher prevalence of hypertension and dyslipidemia. In particular, the higher prevalence of hypertension and trend toward higher use of anti-hypertensive medications may directly affect the BP response during exercise and change of HR. This is a major limitation of our study. Second, this was a retrospective study. Third, the definition of MVA was insufficient, and there was no evidence to suggest ischemia based on other imaging studies such as cardiac MRI or myocardial SPECT. Fourth, although we excluded patients with significant coronary spasm, we could not exclude the effect of microvascular spasms. Fifth, because this study was performed at a single tertiary care center, it is possible that biases existed with respect to patient referral and population sampling. However, we believe the results of this study show a valuable trend in MVA, especially regarding the use of HRR to evaluate this condition.

\section{Conclusion}

Patients with MVA showed reduced HRR and greater EBPR compared with the control group, which suggest that there is a link between MVA and autonomic dysregulation.

\section{Acknowledgements}

Not applicable.

Funding

The authors declared that this study has received no financial support.

Availability of data and materials

Not applicable.

Authors' contributions

K-IC conceived and designed the study. B-JK analyzed the data and wrote the manuscript. E-AJ collected the data. S-II, H-SK and J-HH helped conduct the literature review. K-IC had overall responsibility for the manuscript. All authors gave support to the study and approved the final manuscript.

Ethics approval and consent to participate Not applicable.

\section{Consent for publication}

Written informed consent was obtained from the patients for publication of their individual details and accompanying images in this manuscript. The consent form is held by the authors and is available for review by the Editorin-Chief.

\section{Competing interests}

The authors declare that they have no competing interests.

\section{Publisher's Note}

Springer Nature remains neutral with regard to jurisdictional claims in published maps and institutional affiliations.

\section{Author details}

${ }^{1}$ Division of Cardiology, Department of Internal Medicine, Kosin University College of Medicine, 34, Amnam-dong, Seo-gu, Busan 602-702, Korea. ${ }^{2}$ Convergence Medicine \& Exercise Science Research Institute, Kosin University College of Medicine, 34, Amnam-dong, Seo-gu, Busan 602-702, Korea.

Received: 30 September 2018 Accepted: 14 January 2019 Published online: 01 March 2019

\section{References}

1. Kemp HG Jr. Left ventricular function in patients with the anginal syndrome and normal coronary arteriograms. Am J Cardiol. 1973;32(3):375-6. 
2. Lanza GA, Parrinello R, Figliozzi S. Management of microvascular angina pectoris. Am J Cardiovasc Drugs. 2014;14(1):31-40.

3. Kaski JC, et al. Cardiac syndrome X: clinical characteristics and left ventricular function. Long-term follow-up study. J Am Coll Cardiol. 1995;25(4):807-14.

4. Cannon RO 3rd, et al. Left ventricular dysfunction in patients with angina pectoris, normal epicardial coronary arteries, and abnormal vasodilator reserve. Circulation. 1985;71(2):218-26.

5. Jones E, Eteiba W, Merz NB. Cardiac syndrome $X$ and microvascular coronary dysfunction. Trends Cardiovasc Med. 2012;22(6):161-8.

6. Epstein SE, Cannon RO 3rd. Site of increased resistance to coronary flow in patients with angina pectoris and normal epicardial coronary arteries. J Am Coll Cardiol. 1986;8(2):459-61.

7. Best $S A$, et al. Heart rate recovery after maximal exercise is blunted in hypertensive seniors. J Appl Physiol (1985). 2014;117(11):1302-7.

8. Kline $C E$, et al. Blunted heart rate recovery is improved following exercise training in overweight adults with obstructive sleep apnea. Int J Cardiol. 2013;167(4):1610-5.

9. Imai K, et al. Vagally mediated heart rate recovery after exercise is accelerated in athletes but blunted in patients with chronic heart failure. J Am Coll Cardiol. 1994;24(6):1529-35.

10. Cole $C R$, et al. Heart-rate recovery immediately after exercise as a predictor of mortality. N Engl J Med. 1999;341(18):1351-7.

11. Miyai $\mathrm{N}$, et al. Exercise BP response in subjects with high-normal $\mathrm{BP}$ : exaggerated blood pressure response to exercise and risk of future hypertension in subjects with high-normal blood pressure. J Am Coll Cardiol. 2000;36(5):1626-31.

12. Thanassoulis $\mathrm{G}$, et al. Relations of exercise blood pressure response to cardiovascular risk factors and vascular function in the Framingham heart study. Circulation. 2012;125(23):2836-43.

13. Weiss $S A$, et al. Exercise blood pressure and future cardiovascular death in asymptomatic individuals. Circulation. 2010;121(19):2109-16.

14. Stewart KJ, et al. Exaggerated exercise blood pressure is related to impaired endothelial vasodilator function. Am J Hypertens. 2004;17(4):314-20.

15. Kayrak $M$, et al. Exaggerated blood pressure response to exercise--a new portent of masked hypertension. Clin Exp Hypertens. 2010;32(8):560-8.

16. Lanza GA. Cardiac syndrome $X$ : a critical overview and future perspectives. Heart. 2007;93(2):159-66.

17. Levy D, et al. Echocardiographic criteria for left ventricular hypertrophy: the Framingham heart study. Am J Cardiol. 1987;59(9):956-60.

18. Bruce RA, et al. Seattle heart watch: initial clinical, circulatory and electrocardiographic responses to maximal exercise. Am J Cardiol. 1974; 33(4):459-69.

19. Gibbons RJ, et al. ACC/AHA 2002 guideline update for exercise testing: summary article. A report of the American College of Cardiology/American Heart Association task force on practice guidelines (committee to update the 1997 exercise testing guidelines). J Am Coll Cardiol. 2002;40(8):1531-40.

20. Akasaka T, et al. Comparison of coronary flow reserve between focal and diffuse vasoconstriction induced by ergonovine in patients with vasospastic angina. Am J Cardiol. 1997;80(6):705-10.

21. Zaya M, Mehta PK, Merz CN. Provocative testing for coronary reactivity and spasm. J Am Coll Cardiol. 2014;63(2):103-9.

22. Lanza GA, et al. Relationship between myocardial 1231metaiodobenzylguanidine scintigraphic uptake and heart rate variability in patients with syndrome X. Ital Heart J. 2000;1(3):221-5.

23. Kannankeril PJ, et al. Parasympathetic effects on heart rate recovery after exercise. J Investig Med. 2004;52(6):394-401.

24. Javorka $\mathrm{M}$, et al. Heart rate recovery after exercise: relations to heart rate variability and complexity. Braz J Med Biol Res. 2002;35(8):991-1000.

25. Takeno $\mathrm{K}$, et al. Masked hypertension, endothelial dysfunction, and arterial stiffness in type 2 diabetes mellitus: a pilot study. Am J Hypertens. 2012; 25(2):165-70.

26. Verdecchia P, et al. Short- and long-term incidence of stroke in white-coat hypertension. Hypertension. 2005;45(2):203-8.

27. Filipovsky J, Ducimetiere P, Safar ME. Prognostic significance of exercise blood pressure and heart rate in middle-aged men. Hypertension. 1992; 20(3):333-9.

28. Lanza GA, et al. Autonomic changes associated with spontaneous coronary spasm in patients with variant angina. J Am Coll Cardiol. 1996;28(5):1249-56.

29. Watanabe T, et al. Circadian variation of autonomic nervous activity in patients with multivessel coronary spasm. Jpn Circ J. 2001:65(7):593-8.

Ready to submit your research? Choose BMC and benefit from:

- fast, convenient online submission

- thorough peer review by experienced researchers in your field

- rapid publication on acceptance

- support for research data, including large and complex data types

- gold Open Access which fosters wider collaboration and increased citations

- maximum visibility for your research: over $100 \mathrm{M}$ website views per year

At BMC, research is always in progress.

Learn more biomedcentral.com/submissions 